\title{
Philosophical Interpretations of Relativity Theory: 1910-19301
}

\author{
Klaus Hentschel \\ University of Göttingen
}

\section{Introduction}

This paper is a summary of my doctoral dissertation on philosophical interpretations of Einstein's special and general theories of relativity, submitted to the Dept. for History of Science, Univ. of Hamburg, in 1989, which was recently published in the Series Science Networks at Birkäuser. ${ }^{2}$ After a brief overview of its content I will focus on a discussion of the method employed to analyse philosophical interpretations of a physical theory.

My analysis is based

- firstly on about 2500 contemporary published texts about the theories of relativity written both by scientists and philosophers. These texts have not been of particular interest to historians or philosophers of science up to now; this is understandable from the fact that many of them contain gross oversimplifications, misinterpretations and incorrect statements about the theories of relativity. But I claim that it is just these misinterpretations that can serve as a clue to a better understanding of the general process by which philosophical interpretations are formed (see next section).

- Secondly, collections of the many unpublished documents preserved in the estates of physicists of that time were another very important source, most notably, the duplicate files of the 'Einstein Archive' at the Hebrew University of Jerusalem located at the Collected Paper of Albert Einstein in Boston, which contain hundreds of letters by philosophers asking Einstein to explain features of his theories. These include Bergson, Bridgman, Cassirer, Metz, Meyerson, Petzoldt, Reichenbach, Schlick among others.

- Thirdly, other unpublished materials of interest were found in the estates of philosophers, e.g., of Reichenbach and Carnap (Pittsburgh), of Schlick (Amsterdam), of Bavink (Bielefeld), of Petzoldt (TU Berlin), or of Friedrich Adler (Vienna). All these letters are very telling about the different strategies used by each philosopher in attempting to understand a particular aspect in the theory to be interpreted. This struggle for a closer understanding of scientific matters outside the traditional realm of philosophy was largely omitted or hidden in the published papers.

PSA 1990, Volume 2, pp. 169-179

Copyright $(1991$ by the Philosophy of Science Association 


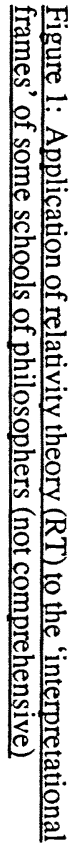

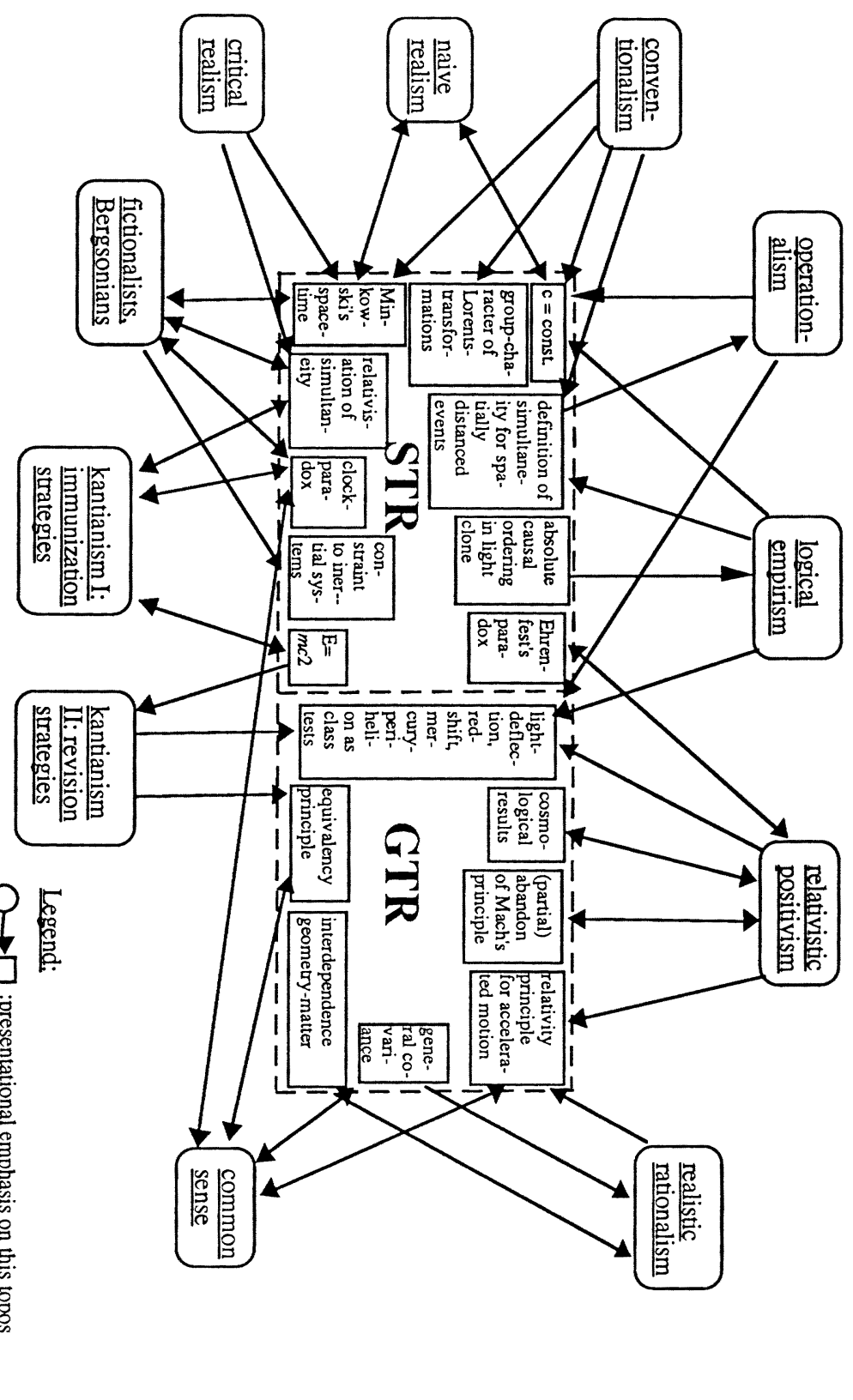




\section{Some General Traits of Philosophical Interpretations}

In figure 1 (= figure 6.1. of my thesis (footnote 1 ).), some (but by no means all) contemporary philosophical schools [ovals] are depicted as they relate [arrows] to selected issues in the theories of relativity [boxes in the center]. Although I could not, of course, achieve completeness with this two-dimensional sketch, it illustrates some general features of philosophical interpretations:

- The various schools discussed the special (left) and general (right) theories of relativity electively, picking out and emphasizing drastically different parts.

- While several groups only dealt with a very few isolated topics from both theories but showing no interest in their context, some schools were ambitious enough to try to incorporate substantial parts of the theories into their interpretations.

- Representatives of some groups of philosophers tried only to understand and to highlight parts of Einstein's theories, while others did not confine themselves to this restricted role, but openly refuted parts of both theories as being in conflict with their general opinions (these cases are marked with double arrows in fig. 1). Although you can often find psychological reasons stemming from the individual personalities of the interpreters behind these different reactions to a compelling scientific issue (an arrogant Oskar Kraus versus a modest Ernst Cassirer), there are also different models about the relationship between physics and philosophy which ultimately account for these astonishing variations. For example, a critique of scientific results was forbidden for the logical empiricists; they could only legitimately analyze them, put them into a broader context and, perhaps, make them thereby more comprehensible to the public. On the contrary, a realm of fundamental issues existed for the neo-Kantians, that could only be approached using a transcendental form of philosophy, which sought to deduce the a priori patterns of the world excluding any possible interference with empiricial issues. Since space and time were among them, a conflict with the special theory of relativity (STR) was to be expected.

\section{The Guiding Idea of My Analysis and Presentation: The Dependence of Philosophical Interpretations on a Framework of Underlying Assumptions}

Now let me briefly discuss how the various philosophical interpretations of relativity theory came about. The issues involved in the special and general relativity theories, which were essentially spelled out since 1905 and 1915, resp., intrinsically involved what could be called an interpretational framework consisting of general epistemological and methodological convictions which ultimately determined the resulting philosophical interpretations of the theories. The vast differences in these often implicit background assumptions explain the formation of completely different, in a sense, incommensurable interpretations of one and the same physical theory.

Some scholars (e.g., John Stachel, Arthur Fine, and at the meeting in Minneapolis, David Stump) have remarked that it is misleading to speak of 'one' theory. Of course, I do not contest that there were a lot of theoretical alternatives to Einstein's general theory of relativity (GTR), construed by Abraham, Nordström, Weyl, Mie, etc., all differing on specific points from the standard general theory, and I also agree that certain points were not resolved for a long time (e.g., value of the cosmological constant, preference of cosmological solutions, singularities). These clearly defined identifiable theoretical alternatives to Einstein's GTR might also deserve a philosophical interpretation in their own right, but their existence does not preclude us from speaking of a standard GTR as physicists do, e.g., in their textbooks on the special or the general theory of relativity. Despite what might be called the not yet settled boundary of the theory ('Forschungsfront', 'research frontier'), where contemporary physicists were in disagreement on many points, what formed the 'hard core' of the theory was undis- 


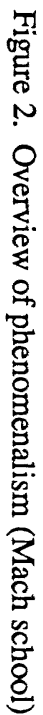

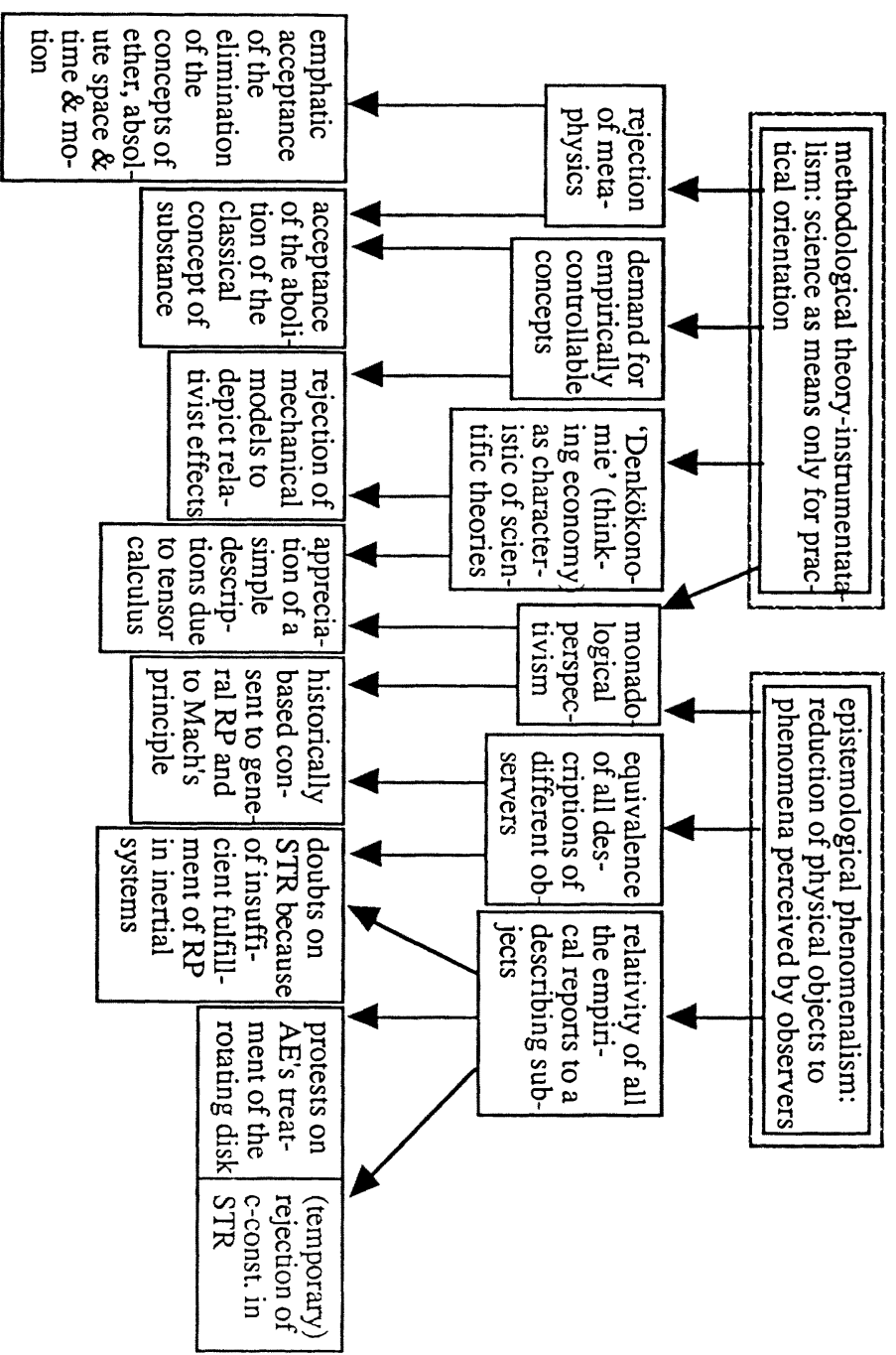


puted and was not altered by further developments at the research frontier. ${ }^{3}$ So for example, the two axioms of the constancy of light in vacuo and the principle of relativity form the 'hard core' of the SRT. In this sense, my model assumption of one theory being interpreted in different ways is justified at least pragmatically. At the end of my paper another argument for this model assumption will be given, based on considerations of what would result if one would not make it.

To get a clear picture of the complicated relationship between general but often implicit background assumptions and specific interpretative statements, between internal and surface structures, to employ the terms of linguists, I modelled the arrival upon an interpretation as a multilevelled process of specialization that I will discuss now within the Machianism example 4 (Cf. fig. 2).

In the beginning of this multigrade process of interpretation we find very few fundamental premises (for the Machians, those of epistemological phenomenalism, coined by Berkeley as 'esse est percipi', and methodological theory instrumentalism). Those basic convictions are obligatory and not questioned by members of this group of philosophers: They form a core (analogous to Lakatos's hard core in scientific theories).

General statements are derived from these core premises in a first specialization step, e.g., about the aim of scientific theories, the status of their results, etc. [see the arrows from the top of figure 2 to the second level]. These general opinions form the level of understanding, so to speak, on which the scientific theory to be interpreted is projected; they define what I earlier referred to as the 'interpretational framework'. In a further step of specialization, the concrete interpretational statements about relativity theory are derived from this interpretational framework [arrows from level 2 to level 3 of figure 2]. The latter is usually fixed before the interpreters started their study of relativity theory. Described in another way (as seen from what will be discussed ahead), those topoi in the theories that offered some connection to their prefixed interpretational framework were selected for further interpretation; others, that did not fit were either left out or reformulated so that they would fit within the procrustean bed. This mechanism already explains the astonishing similarities in the selections of independent interpreters from similar backgrounds.

Let us regard our example. Again and again the Machians emphasized, that for them the physical principle of relativity, one of the axioms of special relativity, was nothing but a trivial consequence of a much broader 'epistemological relativity' between a describing subject and the resulting description. For Petzoldt, whose own philosophical system was consequently called "relativistic positivism", this "relativity' of motion, of size, of time (and of virtually everything else) was the central feature of both theories of relativity - he largely ignored its complementary aspect, namely the prescript of finding observer-independent invariants.

And it is in this area of obliterated parts of the theory where the defects of his interpretations show up. Not only Petzoldt, but all Machians had serious trouble with an adequate understanding of the postulate of the constancy of light $c$ in vacuum, the second axiom of special relativity. You can often find their claim in letters to Einstein and other physicists, but also in their publications, that a properly understood theory of relativity should not contain absoluta independent of observers. They demanded the complete relativization of all physical quantities, thereby completely misunderstanding the aim of Einstein's relativity theory, ${ }^{5}$ and they openly rejected an absolute magnitude like c. ${ }^{6}$ The Machians operated for a couple of years with this obviously philosophically motivated pseudo-argument against the special theory of relativity, 
until Petzoldt and some of his allies (e.g., Lämmel) found a way to make their peace with the constancy axiom of special relativity via their methodological demand of simplicity. This step required a sort of reordering of their hierarchy of norms (simplicity of the axiomatically formulated theory now ranked above proper relativization, formerly it was the other way round), but this example demonstrates once again, that inspite of considerable mental work it was possible to adapt the interpretative framework to the challenges of a new scientific theory, if and only if one was only willing to revise this framework instead of artificially remolding the theory to their own guidelines. So much for the Machian example.

Many examples and case studies in my thesis show the following bias in philosophical interpretations of physical theories. On the one hand, most of the interpreters of relativity theory highlighted and clarified at least one of its facettes, thereby helping in the understanding of its meaning and of its context, or brought to light historical roots of some of the steps made in it. Let me give you some examples: Bridgman's operationalism focussed on Einstein's operational definition of simultaneity for spatially distant events; Cassirer's form of neo-Kantianism emphasized the conceptual change in the constitution of physical objects - away from the classical (material) substances, towards formal (mathematical) invariants of tensor calculus. Poincare and the later conventionalists helped to realize the existence and to understand the significance of free conventions, e.g., in the description of the geometry of space; logical empiricists further clarified the complicated relationship between theoretical terms and observational statements, rules of correspondence and various layers of theories. Several groups also proposed reasons why Einstein's relativity theories were preferable to their historical alternatives.

On the other hand, most of the competing contemporary interpreters of relativity theory fell into the all too human mistake of overstressing their point, e.g., Bridgman's operationalism fit well for an understanding of Einstein's redefinition of simultaneity, but did not help in understanding the meaning of Christoffel symbols of the second kind or the Ricci tensor. It was a mistaken view of the meaning of relativity theory that led Heisenberg to expose the view (in a discussion with Einstein), that it was the moral of relativity theory to use only operationally definable concepts. ${ }^{7}$ In fact, many of the contemporary philosophers unfortunately did not even reach an adequate level of understanding of the factual content of statements derived from the theory; some of them even ended up in open conflict with it by contradicting some relativistic results caused by their disharmony with philosophically inspired assumptions, for example, the Bergsonians and their insistence upon the uniqueness of time as ultima ratio of life and consciousness, or Dingler and his unshakable belief in the euclidity of space, that he regarded as proven once and for all by his method of exhaustion. Very often in cases of conflict, the philosophical presuppositions were not reconsidered or questioned, but rather the scientific theory, irrespective of all scientific motivations for it. Either the theory was declared to be incompetent for dealing with matters reserved to philosophers (as the conservative neo-Kantians said with respect to pure space and time as opposed to their empirical measurement which they held to be the task of physics), or the value and truth of the theory was radically questioned. ${ }^{8}$

4. Further Application of this Method: The Formation of a Protective Belt of Einstein Defenders and Resulting Incommensurability in Philosophical Debates on the Theories of Relativity

The method of setting apparently independent interpretative statements within an interpretative framework that explains how they came about, how they were motivated, is not only useful for a better understanding of the merits and limits of individual inter- 
pretations, but is also helpful in bringing to light interesting consequences that appeared in the discussions of competing groups of philosophers about their resp. interpretations.

One such consequence was the formation of what I have called a 'protective belt' of defenders of both theories of relativity - see figure 3 (=fig. 3.1. of my thesis). By 1920 , Einstein had become tired of repeatedly countering the antirelativists with the same arguments. So, philosophers with strong pro-relativistic opinions like

Reichenbach and Petzoldt took over the defense of his theories, by inevitably evoking norms and arguments stemming from their own interpretational frameworks. Now the Einstein opponents responded to these defenses by confusing relativistic statements with the corresponding philosophical interpretations by Einstein's defenders. For in-

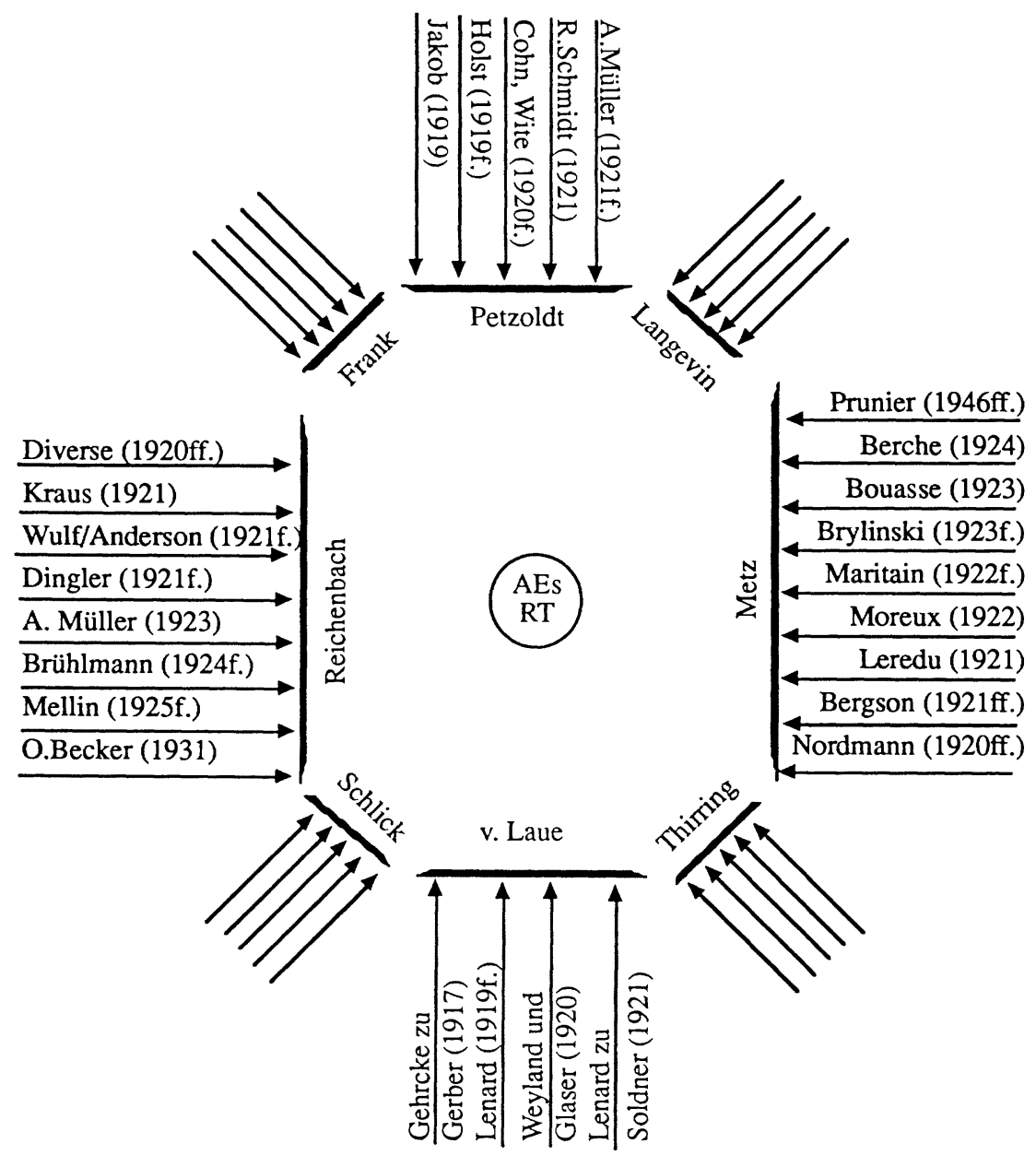

Figure 3. Overview on the contemporary disputes about relativity theory and the most pertinent defenders of Einstein 
stance, Petzoldt used his monadological perspectivism to defend the special theory of relativity against charges of missing absolutes and thereby provoked a further and more serious misunderstanding of this theory as subjectivism and relativism, which Einstein clearly had not meant.

An even more radical form of misunderstanding occurred in most contemporary discussions between members of different groups. Here different interpretational frameworks clashed with each other. Both parties usually thought they had found compelling arguments to defeat their opponents but felt misunderstood by the other side, whose arguments simply seemed to go pointlessly astray. These heated discussions, full of metaphors of strife and battle, ${ }^{9}$ were subsequently forgotten but they are a highly interesting area for incommensurability studies.

\section{Preconditions and Criteria for a Successful Interpretation}

As mentioned before, I did not simply want to discuss all the historic cases of successful or unsuccessful interpretations, but also to find out what could be learnt from the mistakes made there-Which of these mistakes could have been avoided and how? In this context, only those cases where something went wrong and misinterpretations resulted were important. The abundance of contemporary interpretations of Einstein's theories of relativity between 1910 and ca. 1930 helped me to formulate the following preconditions for sound interpretations that avoid sidetracks, such as the distortion of the idea or statement at hand:

1. Interpreters should show modesty and open-mindedness-it is they who are walking on foreign territory, so they have to learn about the procedure, the rules of argumentation, and the scientific facts and laws to be obeyed.

Although it sounds quite trivial, this precondition was by no means fulfilled in the case of Einstein's interpreters. ${ }^{10}$

2. Philosophical interpreters should aim at a precise understanding of the technical, mathematical language, in which the theory is formulated. Only then can they really understand its structure; only then can they get a feeling for the strength of intratheoretical derivations and for the harmony (or as scientists often say: the beauty) of it as a whole. Otherwise, a partial, fragmented understanding is the unavoidable result, in which some axioms, theorems or statements are overemphasized while others are illegitimately ignored. The selectivity of most contemporary interpretations is a clear indication of the inobservance of this precondition. Also, the overwhelming majority of the most accurate interpreters were not accidently educated physicists or mathematicians, i.e., Schlick, Reichenbach, Frank, Eddington, Weyl, Metz, Bavink.

3. If conflicts arise between the interpretational framework and some results of the scientific theory being interpreted, there should be a willingness to revise these underlying philosophical assumptions, that is, to accomodate the traditional philosophical outlook to the new challenge. Although philosophers seem to be very conservative in the revision of traditional concepts and assumptions, the examples of Cassirer, Elsbach, Winternitz and of the early Reichenbach show, that all of them were willing to revise the Kantian interpretative framework about how to apply the transcendental method and what to count as an a priori condition for human experience. Consider their far-reaching concessions in contrast to the majority of Kantians who insisted on the rigid adherence to their basic premises, immunizing themselves against any possible refutation by scientific results. (Natorp, Hönigswald, Sellien, etc.). ${ }^{11}$ 
4. Philosophical interpretations are not the right place for a critique of the methods, goals or results of scientific theories, because only scientific criteria decide about their adequacy in scientific discourse. Philosophers do not have to judge about the accuracy of theories. Philosophers do not have to judge about the accuracy of theories, but they can help to make them more accessible to nonscientists by analysing their content and their methods and putting them into broader context ("Wissenschaftsanalyse" in the sense of Reichenbach).

5. In order to a avoid unbalanced presentations and to avoid the danger of only noticing what is in accordance with one's own prefixed interpretational framework, 12 it is very helpful to study competing interpretations made from a very different perspective, even if one doesn't agree with them. It is by no means an accident, that the richest interpretations were made by Reichenbach and Metz, both philosophers who very actively discussed all kinds of aspects of relativity theory with proponents of virtually all the other contemporary schools. ${ }^{13}$ All these disputes helped them to overcome the natural tendency toward a one-sided interpretation.

6. When the previously mentioned preconditions are fulfilled, the crudest forms of misinterpretation can be avoided. But still, there will remain a large spectrum of competing interpretations of one and the same theory. It is useful to have some criteria for a comparison of these interpretations. I propose the following ones:

Breadth: The total amount of scientific material incorporated into the interpretation. Compare the difference in quality between Reichenbach's Philosophie der Raum-Zeit or Meyerson/Metz's accounts of la Relativité against the lean traces of relativity theory in the writings of Natorp, Driesch, Brunschvicg and many others.

Depth: Only a few authors tried to include more complicated themata in their interpretations, for example, Reichenbach's discussion of the repetition of the Michelson-Morley experiments, Metz's clarification of the meaning of relativistic length contractions, Eddington's idiosyncratic but at least stimulating treatment of the field equations of general relativity in his Space, Time, and Gravitation.

Currentness: Only a few interpreters really understood those fields that were still under debate among scientists of the time, for example, the issue of singularities in general relativity, the debates about different models of cosmology, and also Russell's vision of a coordinate-free form of general relativity.

Historical context: E.g., Cassirer's well-founded claim that Einstein's relativity theory constitutes a further stage in the gradual substitution of material substances with mathematical forms, Meyerson's original, but debatable thesis of Hegelian traces in Einstein's Déduction Relativiste).

Naturalness of interpretation: That is, avoidance of conceptual gymnastics to accommodate the factual content (Reichenbach, Schlick, Cassirer), and finally

Uniformity and harmony in presentation (Cassirer, Schlick, Meyerson, Reichenbach in his axiomatics). 
Among the contemporary interpretations of the theories of relativity, Hans Reichenbach fulfilled all preconditions and most of the criteria in the sixth point above. Schlick's interpretation, preferred by Einstein between 1915 and 1925, was too cursory-his early violent death prevented a more detailed account from his pen. Meyerson's interpretation, favored by the later Einstein, and Metz's later contributions very much in the spirit of Meyerson, worked as a sort of complementary interpretation focussing on points underestimated by the logical empiricists. But in toto it was certainly much less exhaustive and broad than Reichenbach's œuvre on relativity-mainly on special relativity. The general theory of relativity doesn't seem to have received an equally balanced treatment by contemporaries-its complexity was only fairly appreciated much later, e.g. in writings of A. Grünbaum, M. Friedman or C. Ray (to name just a few).

It is only by giving this set of criteria to compare the quality of competing interpretations, that interpretational relativism can be avoided. In my opinion, these main criteria (perhaps among others, less trivial and not yet formulated ones) allow us to treat competing interpretations of scientific theories in the same objective, rational way in which philosophers of science have tried to deal with competing scientific theories for many decades. I am well aware of the fact that the preconditions and criteria listed above will be as controversial as the analogous proposals for criteria of theory evaluation have been. But at least they might trigger the beginning of a discussion about comparative evaluations of philosophical interpretations of scientific theories. This should, in the light of the ongoing discussions about the philosophical interpretation of quantum mechanics, quantum field theories, etc., be an important topic on the agenda of philosophers of science.

\section{Notes}

1First of all, many thanks for the invitation to the organizers of the PSA meeting and to Prof. Don Howard (Univ. of Kentucky, Lexington), who had initiated the Colloquium on "Recent Work in the History of the Philosophy of Science". My dissertation was supervised by Prof. Dr. Andreas Kleinert (Institut für Geschichte der Naturwissenschaften, Hamburg) and Prof. Dr. Lothar Schäfer (Philosophisches Seminar, Univ. Hamburg). The editors at the Collected Papers of Albert Einstein in Boston and many archivists and librarians elsewhere have supported my research. Miss Ann M. Lehar helped me a lot in improving my English for the written version of this paper.

2Interpretationen und Fehlinterpretationen der speziellen und der allgemeinen Relativitätstheorie durch Zeitgenossen Albert Einsteins, Birkhauser, Basel, 1990; ca. 800 pp., more than 3000 bibliographic entries, many figures and tables.

${ }^{3}$ See also Chr. Ray: The Evolution of Relativity, Hilger, Bristol, 1987, for a similar view of relativity theory.

4See also section 4.8. of my thesis (fn. 2).

5 Einstein later reflected about alternative names, such as 'theory of absoluta' or 'theory of invariants' to distinguish his theory from what was commonly refered to as 'relativism' - see sect. 2.4. of my thesis (fn.2). 
6When Einstein made $\mathrm{c}$ a variable dependent on the gravitational potential in his Prague theory of 1911, several Machians (and I would also expect Mach himself) regarded this (erroneously) as a fulfillment of their demand of rigorous relativization.

${ }^{7} \mathrm{~A}$ recipe that Heisenberg later tried to implement in quantum mechanics.

${ }^{8}$ Vaihinger's pupils declared it as merely a convenient fiction; Dingler's certicism repeatedly declared relativity theory as stillborn and portrayed himself in the role of a high priest ringing its death knell; see sections 4.4. and 4.5.4. of my thesis. (fn.2).

${ }^{9}$ In my thesis, I treated the discussion between Kraus, Urbach and Frank in more detail (sect. 5.3.) and I also studied one person (Reichenbach) in the context of a multiple front war (sect. 3.4.3.). But other figures such as Dingler and Reichenbach are worth studying in more detail.

10For example, the neo-Kantian journalist Drill wrote angrily in reply to Max Born: "I'm not willing to talk about Einstein and I'm not competent to do so. [...]. But nobody has to accept it, when a scientific theory tries to cheat common sense, whoever may have formulated it." And many professional philosophers did not react much better.

11 The Kantian Drill emphatically wrote against the reformist wing: "A philosopher should know that physics can't lead the way for philosophy. It is the latter's task to find out the necessary conditions for all knowledge, including all sciences."

12 Somehow according to the principle: one sees what one expects to see. Note that theory-ladenness of observation was emphasized by Duhem and Quine!

${ }^{13}$ See sect. 5.1. and 5.2 of my thesis. 